\title{
Malades et maladies dans l'espace public
}

\author{
Benoît Lafon et Isabelle Pailliart
}

\section{OpenEdition}

Journals

Édition électronique

URL : http://journals.openedition.org/questionsdecommunication/7319

DOI : 10.4000/questionsdecommunication.7319

ISSN : 2259-8901

\section{Éditeur}

Presses universitaires de Lorraine

\section{Édition imprimée}

Date de publication : 1 juillet 2007

Pagination : 7-15

ISBN : 978-2-86480-829-9

ISSN : 1633-5961

\section{Référence électronique}

Benoît Lafon et Isabelle Pailliart, « Malades et maladies dans l'espace public », Questions de communication [En ligne], 11 | 2007, mis en ligne le 01 juillet 2007, consulté le 12 avril 2021. URL : http://journals.openedition.org/questionsdecommunication/7319; DOI : https://doi.org/10.4000/ questionsdecommunication.7319 


\title{
$>$ DOSSIER
}

\author{
BENOÎT LAFON
}

Groupe de recherche sur les enjeux de la communication

Université Stendhal-Grenoble 3

benoit.lafon@u-grenoble3.fr

ISABELLE PAILLIART

Groupe de recherche sur les enjeux de la communication Université Stendhal-Grenoble 3 isabelle.pailliart@u-grenoble3.fr

\section{MALADES ET MALADIES DANS L'ESPACE PUBLIC}

S

'interroger sur la place des malades dans l'espace public et sur les modalités de visibilité et de construction des « maladies » nécessite d'opérer un déplacement. En mettant l'accent sur les « malades » plutôt que sur les autres acteurs du secteur de la santé (les scientifiques, les chercheurs, les médecins, les spécialistes...), l'objectif est de prendre en compte et d'analyser en profondeur plusieurs évolutions: l'exposition de la vie privée, la place du témoignage, la « professionnalisation » des patients... qui tendent à remettre en cause les fonctionnements traditionnels de ce secteur.

Les institutions hospitalières ont amorcé un mouvement de reconnaissance des malades en les impliquant dans leurs structures, les pouvoirs publics ont fait du patient « l'acteur de sa santé », les associations de malades jouent un rôle de plus en plus important dans la diffusion d'informations, dans la défense des intérêts de ceux-ci ou dans les actions de 
prévention à destination de ceux-ci. Ce mouvement est récent. Dans le domaine de la santé tel qu'il se construit publiquement, les malades ont, jusqu'à l'épidémie de sida, disposé d'une place marginale malgré leur statut central. Aujourd'hui, comme le note Janine Barbot (2002 : 28), les malades et leurs associations « ont changé » au point que « l'activisme thérapeutique a eu pour enjeu et pour conséquence de participer à la redéfinition des frontières entre science et espace public ». En particulier dans le cas du sida (Barbot, 2002 ; Paicheler, 2002 ; Dodier, 2003), la dernière décennie marque l'apparition des associations de malades avec lesquelles le corps médical doit discuter. Sur ce point, les chercheurs identifient la diversité des formes d'engagement, qu'elles soient associatives ou « ordinaires », qu'elles aient pour objectif de rendre le patient « actif » dans la gestion de sa maladie ou de déboucher sur un « activisme thérapeutique » mettant en œuvre « de nouvelles formes de collaborations avec les agences publiques »(Barbot, 2002 : 28I). Ainsi les observateurs remarquent-ils l'apparition progressive d'une expertise associative, de même que, au fur et à mesure des recherches menées, sont abordés les positionnements évolutifs des associations visà-vis du milieu médical. Dans le cadre du sida, « l'offensive » des associations et le rôle des journaux dans leur reconnaissance publique a contribué à produire « un désenclavement du monde spécialisé des essais contrôlés » (Dodier, 2003 : 204). Cependant, il n'est pas certain que ces évolutions soient généralisables à d'autres maladies. De ce point de vue, la question du cancer abordée dans cette livraison de Questions de communication semble à l'opposé de celle du sida. Les associations présentes dans le secteur le sont depuis de nombreuses années (la Ligue contre le cancer fut créée en 1918), elles ont à leur tête des médecins ou des hauts fonctionnaires et, sur le long terme, ont été relativement proches du milieu médical, les passerelles entre les malades et les médecins étant en quelque sorte médiées par ces associations. Du même coup, la place des malades est restée contrôlée et leur représentation dépendante du pouvoir médical.

Par ailleurs, le secteur de la santé se caractérise par un poids tout particulier de l'expertise médicale et de la technicisation des savoirs, ainsi que par une marchandisation des dispositifs, une spécialisation des journalistes, et une implication des pouvoirs publics nationaux et locaux, qui rendent absentes les conditions habituellement requises pour la constitution d'espace public (formation et circulation des opinions, expressions de points de vue contradictoires, confrontations et rapports de force ...) qui conduit à penser la sphère publique plus en terme « d'évitement de la dimension conflictuelle des débats » (Dodier, 2003 : 264) qu'en terme de « démocratie sanitaire ». Ainsi la notion de maladie renvoie-t-elle à la lutte des acteurs pour imposer les cadres de lecture d'une réalité sociale et de sa construction. L'accès à l'espace 
public d'une maladie comprend donc de lourds enjeux politiques (dans la mise en œuvre d'une politique publique de santé par exemple) : a contrario, cette dimension est illustrée par l'hépatite C dont, en France, « I'histoire du cheminement est celle d'une maladie sans véritable identité » (Jouzel, Landel, Lascoumes, 2005 : 203), alors que les situations étrangères indiquent une visibilité de l'épidémie dans les différents espaces publics nationaux.

S'inscrivant dans ce contexte, trois thèmes sont plus particulièrement abordés par les contributeurs de ce dossier : les questions de santé au sein de l'espace public sociétal, la production de l'information et ses contraintes, la relation individuelle et sociale à la maladie.

\section{Le champ des médias : un enjeu pour contrôler l'espace public}

Le thème de la santé présente deux types d'espaces : un espace public sociétal et un espace spécialisé et offre, de ce fait, l'opportunité d'étudier leur confrontation. Le premier est, en grande partie, animé par les médias et la presse d'information générale, le second l'est par les médias spécialisés propres à la communauté scientifique. L'étude des relations entre ces deux espaces - l'espace public sociétal et l'espace public scientifique - illustre les tentatives du second pour maîtriser le premier. L'enjeu reste le contrôle par les scientifiques de l'espace public sociétal et cela sous différentes formes: encadrement des prises de paroles, discours d'autorité, maîtrise de l'information. Les informations médicales dans les médias sont particulièrement révélatrices de ce poids des institutions scientifiques. Le plus souvent, la caution apportée par une publication préalable dans une revue scientifique constitue une condition indispensable à une diffusion dans les médias, du moins la légitimité des modalités de sélection des articles scientifiques retenant l'attention des journalistes.

Le champ des médias est un enjeu pour le contrôle de l'espace public. D'ailleurs, une partie des critiques des scientifiques vis-à-vis des médias et des journalistes peut se lire comme un refus de l'autonomie de ce champ, le dénigrement s'inscrivant dans une stratégie de conservation de pouvoirs. Ces éléments ont été mis en valeur par Nicolas Dodier (1999) à travers les urgences scientifiques et « l'affaire de la ciclosporine ». Ce dernier montre les oppositions entre institutions scientifiques et champ des médias, même si des clivages existent à l'intérieur de chacune de ces catégories (ibid. : 137). Ainsi l'entrée dans l'espace public est-elle bien un enjeu. Elle correspond à une « lutte menée par les institutions éthiques 
et scientifiques consacrées contre une circulation de l'information non contrôlée par les revues scientifiques [qui] marque la vigueur très actuelle du mouvement historique de plus long terme qui vise à encadrer, par les arènes spécialisées, les prises de parole dans la sphère publique » (ibid. : 142). Ainsi la place de la santé dans la sphère publique, renforcée par les actions de prévention, par la presse de vulgarisation médicale, par la transformation des émissions télévisées de santé ou encore par l'essor des sites internet, ne conduit-elle pas automatiquement à la création d'un espace public de la santé. Ce dernier pourrait se rapprocher d'un « espace public scientifique [qui] se trouve éclaté, tiraillé entre des forces divergentes et dépourvu d'instance incitatrice forte et légitime du point de vue de l'animation du débat et de la formation d'une opinion publique » (Miège, in : Pailliart, 2005 : 134).

La contribution d'Isabelle Pailliart et Géraldine Strappazzon, « Les paradoxes de la prévention des cancers : publicisation et privatisation », évoque la question d'une objectivation du malade en montrant qu'au niveau national (présidentialisation) comme au niveau local (territorialisation), les acteurs politiques cherchent dans une large mesure à maîtriser les enjeux communicationnels sur la question. Cette problématique d'un espace public partiel est aux fondements du propos d'Helena Sandberg et Peter Dahlgren, « La construction médiatique de l'obésité dans l'espace public suédois », fondé sur le constat d'une nécessaire collaboration entre médecins, scientifiques et journalistes pour voir émerger un réel espace public, aujourd'hui absent. Pour sa part, dans «La santé à la télévision : émergence d'une question sociale », Hélène Romeyer traite de l'espace public de la santé en se fondant sur l'évolution des magazines de la télévision française. Montrant le glissement des magazines télévisés vers un modèle social et non plus médical et scientifique, elle met en évidence l'émergence d'une question sociale de la santé.

\section{De l'autonomisation des médias à celle des malades}

Dans leurs tentatives d'accès à l'espace public, les malades rencontrent les attentes de certains médias dont l'objectif est aussi de se dégager de contraintes externes (celles du champ médical) : malades et maladies se heurtent alors aux multiples exigences des médias écrits et audiovisuels. C'est à une réflexion sur ce double mouvement de contraintes réciproques entre, d'une part, médias et champ médical et, d'autre part, médias et malades, que cette livraison invite. Dans le domaine médical, l'autonomisation croissante des médias a notamment été abordée par Patrick Champagne (in : Mathien, 1999). Dans une étude sur « Les 
transformations du journalisme scientifique et médical », ce dernier établit le fort développement et la spécialisation de l'information médicale dans les médias d'information générale au cours des années 80 et 90. Si à partir des années 60, le développement des médias audiovisuels suscite de nombreux débats dans les instances professionnelles représentatives des médecins, l'expansion d'un marché économiquement rentable de l'information scientifique favorise une structuration du groupe des journalistes scientifiques et du rubricage de ce type d'information, au point que de nombreux titres de presse magazine spécialisée sur la médecine et la santé se développent. En 1993, on compte 400 titres de presse médicale. L'apparition du sida sera à la fois un révélateur, un opérateur, et un accélérateur de ce phénomène. Enfin, la santé va devenir l'objet d'une demande d'information critique, favorisée par les demandes pressantes des malades, représentés par des associations actives en ce domaine. Ainsi les médias pèsent-ils aujourd'hui plus fortement dans le fonctionnement d'univers sociaux auparavant très fermés, tel que celui de la santé.

Mais cette ouverture progressive de l'information médicale dans les médias ne se fera pas sans rapports de force. En effet, elle est objet d'affrontement entre, d'un côté, les institutions scientifiques, de l'autre, l'autonomie progressivement acquise des médias et du champ journalistique. En cela, les médias ne jouent pas le rôle de courroie de transmission des autorités scientifiques (ou politiques), et le champ des médias n'est pas le porte-parole de prises de positions et le reflet de champ de forces. La télévision, « en tant qu'institution disposant de ses propres systèmes de valeurs, et composée d'acteurs qui les assument, ne produit pas un décalque des représentations sociales. Si la rationalité fonctionne comme une matrice culturelle, elle ne structure qu'en partie le discours télévisuel : celui-ci dispose d'autonomie »(Babou, 2004 : 154- |55). La question qui se pose au champ scientifique et médical, de même qu'aux malades et à leurs associations, est alors celle de la définition d'une médiatisation conforme à leurs attentes.

La question de l'autonomisation croissante des médias a notamment conduit à traiter de la manière dont les émissions de télévision contribuent à l'instauration sur la scène publique des questions de santé. Le Téléthon en fournit un exemple (Walter, 1998) : en faisant appel à la générosité des individus, ce dispositif favorise des réflexions sur le rôle de l'État, le rapport de l'individu avec le désarroi d'autrui, la monstration de la souffrance, ou encore la place du témoignage. C'est donc sous l'angle des rapports avec le politique que le Téléthon est dans un premier temps traité, la télévision étant vue comme révélatrice de tendances lourdes de la société. Ainsi cet événement « interroge[-t-il] trois éléments clefs du politique (Chambat, 1994) : l'identité collective (l'appartenance à la nation), la participation politique [...], la compétence politique du peuple 
(formation civique : la générosité comme alternative à la revendication, au débat politique; la survalorisation des experts...) » (Walter, $1998: 20)$.

Plus généralement, le rôle des médias est à replacer dans des questionnements sur la construction des problèmes publics, dont ils sont partie prenante. A ce sujet, Emmanuel Henry traitant de l'amiante note bien que la nécessité première pour les journalistes est de « construire le problème de telle sorte qu'il puisse attirer l'attention du public, et justifier ainsi l'attention qu'ils y portent », et ce à l'aide de différents procédés, tels que la construction de ce problème comme « thème récurrent de l'information quotidienne », ou la « redéfinition du risque comme touchant l'ensemble de la population » (Henry, 2000 : 564). Ainsi la «problématisation » de situations, longuement absentes de la sphère publique, conduit-elle à s'interroger sur la place des médias, les stratégies professionnelles des journalistes, et sur les rapports de force entre organisations publiques ou privées avec des associations de défense des intérêts d'une population. C'est le cas du « scandale du sang contaminé » qui se présente comme un fait social construit par les logiques d'acteurs et par leur affrontement. Ainsi « la qualification des faits comme "scandaleux", loin d'avoir été évidente et immédiate, a[-telle] été le résultat d'une lutte singulière qui a notamment opposé, durant de longs mois, certaines victimes de la contamination par le sang à l'État, à la justice, aux journalistes, puis les journalistes aux milieux médicaux et politiques et enfin les journalistes entre eux » (Champagne, Marchetti, 1994 : 43). La construction des problèmes repose donc sur des positionnements propres à chaque champ (politique ou médiatique) et sur la dynamique de ces positionnements.

S'intéressant à « Une rubrique "à part"” », c'est-à-dire à « l'information médicale de l'après-guerre au début des années 80 », Dominique Marchetti note que la médecine était alors un espace clos aux représentations consensuelles, à la différence de la période actuelle, marquée par des processus d'« économicisation », de politisation et de moralisation des enjeux. Cette logique quasi consumériste de la santé est présentée dans l'article de Philippe Ponet: « Les logiques d'une consécration journalistique. L'exemple des "50 meilleurs hôpitaux en France" ». L'auteur y souligne les nouvelles contraintes pesant sur les hôpitaux, sommés de souscrire à une triple logique : logique médiatique de la transparence, logique économique de la maîtrise des dépenses et logique techniciste de la rationalisation des pratiques de soins. Enfin, c'est à l'examen des logiques médiatiques contraignantes que se sont livrées Leila Azeddine, Gersende Blanchard et Cécile Poncin dans « Le cancer dans la presse écrite d'information générale. Quelle place pour les malades? ».. 


\section{Le rapport individuel et social à la maladie}

Le rapport individuel et social à la maladie renvoie à l'idée que le colloque singulier entre le patient et le médecin n'est pas seulement une question de relation et de savoir. Elle ne repose pas seulement sur la capacité du médecin à faire preuve de compétences et d'un sens de la relation. Le rapport des individus à un médecin est également lié au rapport de ceux-ci à l'institution médicale et hospitalière. Le colloque singulier possède donc toujours une dimension politique (renvoyant aux moyens et aux politiques publiques). Ce rapport est social, car il est aussi dépendant des formes de médiation qui existent dans les relations entre le patient et le médecin, d'une part, et entre le patient et la maladie, d'autre part. Ainsi la manière dont les patients recherchent de l'information - dans des journaux, sur des sites internet - auprès d'autres malades contribue-t-elle à la transformation de ces relations avec les professionnels de la santé. Par le biais de publications soutenues par des acteurs associatifs et institutionnels, mais aussi par l'utilisation de réseaux de communication numériques - forums, blogs, listes de diffusion -, les malades de diverses affections prennent la parole. Ce phénomène a notamment été analysé par Madeleine Akrich et Cécile Méadel (2002) dans le cadre d'échanges entre patients sur les médicaments dans des listes de discussion électroniques. Si ces discussions volontaires des patients (par leur inscription préalable) sont une réelle prise de parole, leur évanescence temporelle empêche des formes d'actions collectives structurées. En outre, les thèmes discutés provoquent des critiques plus ou moins acerbes du corps médical : « Si les traitements des tumeurs cancéreuses font l'objet de peu de contestations, il en va différemment en ce qui concerne les "effets secondaires" de la maladie, effets des traitements (nausées, douleur...) mais aussi effets du cancer lui-même (fatigue, angoisses, etc.) 》(Akrich, Méadel, 2002 : 101-102). Ainsi les malades développent-ils une capacité d'expertise dans le traitement de leur maladie et du « vivre avec ». De fait, les individus acquièrent une visibilité tandis que les médecins se voient interrogés sur leur capacité à intégrer les demandes sociales (en se rapprochant de leurs patients). Ces deux tendances sont illustrées dans cette livraison par deux articles, la confrontation distanciée avec le cancer dans les fictions télévisées par Benoit Lafon (« Le cancer en prime time. Émergence d'une confrontation distanciée avec la maladie par les fictions télévisées ») et l'annonce en cancérologie par Antoine Spire et Rollon Poinsot ( $\mathrm{La}$ problématique de l'annonce en cancérologie »). Si le premier article aborde la fiction télévisée comme révélatrice d'une évolution d'un rapport à la maladie (les individus se confrontant de manière croissante à celle-ci par le biais de la feintise ludique), le second montre comment les médecins pourraient replacer les patients au cour de l'action thérapeutique par une interaction adaptée. Enfin, Philippe Bataille évoque 
« La mort, le malade, le proche » comme « Une question de communication entre ego et alter, et autrui » : décrivant comment les malades portent deux fardeaux, « la crainte légitime d'être un jour emporté par la maladie et celle qu'ils suscitent chez les autres », il montre la nécessité d' « admettre que la mortalité fait partie intégrante de l'existence »(Bataille, 2007 : 156).

Finalement, les thèmes abordés sont tous reliés à la notion de gouvernement des hommes et à la « biopolitique » au sens de Michel Foucault (2004: 323) qui l'entend comme « la manière dont on a essayé, depuis le $18^{\text {e }}$ siècle, de rationaliser les problèmes posés à la pratique gouvernementale par les phénomènes propres à un ensemble de vivants constitués en population : santé, hygiène, natalité, longévité, races... ». C'est donc bien sur le temps long qu'il faut situer les transformations en cours, et c'est dans le cadre de la rationalité politique que se pose la question des malades et des maladies dans l'espace public.

\section{Références}

Akrich M., Méadel C., 2002, Sciences sociales et santé, 20, vol I, pp. 89-I I 6.

Azeddine L., Blanchard G., Poncin C., 2007, « Le cancer dans la presse écrite d'information générale. Quelle place pour les malades ? », Questions de communication, I I, pp. I | I- 127.

Babou I., 2004, Le cerveau vu par la télévision, Paris, Presses universitaires de France.

Barbot, J., 2002, Les malades en mouvements. La médecine et la science à l'épreuve du sida, Paris, Balland.

Bataille Ph., 2007, « La mort, le malade, le proche. Une question de communication entre ego et alter, et autrui », Questions de communication, I I, pp. |49- 158.

Champagne P., Marchetti D., 1994, « L'information médicale sous contrainte. À propos du "scandale du sang contaminé" », Actes de la recherche en sciences sociales, I0I-102, pp. 40-62.

Dahlgren P., Sandberg H., 2007, « La construction médiatique de l'obésité dans l'espace public suédois », Questions de communication, II, pp. 33-49

Dodier N., 1999, « L'espace public de la recherche médicale. Autour de l'affaire de la ciclosporine », Réseaux, 95, pp. 107-154.

- 2003, Leçons politiques de l'épidémie de sida, Paris, Éd. de l'École des hautes études en sciences sociales.

Foucault M., 2004, Naissance de la biopolitique, cours au Collège de France, 19781979, Paris, Gallimard/Éd. Le Seuil

Henry E., 2000, Un scandale improbable. Amiante : d'une maladie professionnelle à une « crise de santé publique », Thèse en sciences de l'information et de la communication, université de technologie de Compiègne. 
Jouzel J.-N., Landel D., Lascoumes P., 2005, Décider en incertitude. Les cas d'une technologie à risques et de l'épidémie d'hépatite C., Paris, Éd. L'Harmattan.

Lafon B., 2007, « Le cancer en prime time. Émergence d'une confrontation distanciée avec la maladie par les fictions télévisées », Questions de communication, II, pp. 129-147.

Mathien M., dir., 1999, Médias, santé, politique, Paris, Éd. L'Harmattan.

Marchetti D., 2007, « Une rubrique "à part". L'information médicale de l'aprèsguerre au début des années 80 », Questions de communication, I I, pp. 7I-90.

Paicheler G., 2002, Prévention du sida et agenda politique. Les campagnes en direction du grand public 1987-1996), Paris, CNRS Éd.

Pailliart I., coord., 2005, La publicisation de la science, Grenoble, Presses universitaires de Grenoble.

Pailliart I., Strappazzon G., 2007, « Les paradoxes de la privatisation des cancers : publicisation et privatisation », Questions de communication, I I, pp. 17-31

Ponet Ph., 2007, « Les logiques d'une consécration journalistique. L'exemple des "50 meilleurs hôpitaux en France" », Questions de communication, I I, pp. 9 I - I 10.

Romeyer H., 2007, « La santé à la télévision : émergence d'une question sociale », Questions de communication, I I, pp. 5 1-90.

Spire A., Poinsot R., 2007, « La problématique de l'annonce en cancérologie », Questions de communication, II, pp. 159-176

Walter J., dir., 1998, Le Téléthon. Scène, intérêts, éthique, Paris, Éd. L'Harmattan. 